\title{
Tenascin-R as a Repellent Guidance Molecule for Developing Optic Axons in Zebrafish
}

\author{
Catherina G. Becker, Jörn Schweitzer, Julia Feldner, Thomas Becker, and Melitta Schachner \\ Zentrum für Molekulare Neurobiologie Hamburg, Universität Hamburg, D-20246 Hamburg, Germany
}

To investigate the role of tenascin- $\mathrm{R}$ in nervous system development, we studied axon pathfinding in the developing optic system of zebrafish. Zebrafish tenascin- $\mathrm{R}$ has the same domain structure as tenascin- $\mathrm{R}$ in amniotes. Amino acid sequence identity with human tenascin- $\mathrm{R}$ is $60 \%$. In 3-d-old larvae, tenascin-R mRNA is expressed in scattered cells throughout the periventricular cell layer of the diencephalon and tectum. Tenascin-R immunoreactivity is not detectable in the optic nerve, optic tract, or tectal optic neuropil but immediately borders the optic tract caudally. Reducing expression of tenascin-R in 3-d-old larvae in vivo by injecting morpholinos into fertilized eggs led to excessive branching of the optic tract in $86 \%$ of all injected larvae compared with $20-37 \%$ in controls. Branches were almost exclusively caudal, where tenascin- $\mathrm{R}$ immunoreactivity normally borders the optic tract, suggesting a role for tenascin- $\mathrm{R}$ in guiding optic axons in the ventral diencephalon by a contact-repellent mechanism.

Key words: extracellular matrix; chondroitin sulfate proteoglycans; semaphorin; slit; axon guidance; Danio rerio

\section{Introduction}

Growing axons are guided to their targets via positive and negative cues. The diversity of axon-repellent molecules of the extracellular matrix, which keep growing axons away from inappropriate territories, is increasingly recognized (for review, see Dickson, 2002). The extracellular matrix molecule tenascin-R, which is expressed by neurons and oligodendrocytes in the adult CNS, has been known for some time to have axon-repellent properties in vitro (for review, see Schachner et al., 1994; Jones and Jones, 2000). For example, neurites of adult retinal explants (Becker et al., 2000), dorsal root ganglion neurons (Taylor et al., 1993), and cerebellar neurons (Pesheva et al., 1993) are repelled by a substrate border of tenascin-R in vitro. Here we describe for the first time a repellent function of tenascin- $\mathrm{R}$ in the guidance of optic axons in vivo.

\section{Materials and Methods}

Animals. Adult (body length, $>2 \mathrm{~cm}$; age, $>4$ months) zebrafish were kept at a 14/10 hr light/dark cycle and were fed dried fish food and live brine shrimp. Embryos were collected from our breeding colony and staged in hours after fertilization at standard temperature $\left(28.5^{\circ} \mathrm{C}\right)(\mathrm{Kim}-$ mel et al., 1995).

Molecular cloning. To isolate the zebrafish tenascin-R cDNA, we performed degenerate reverse transcription-PCR with oligonucleotide primer sequences [P1, 5' -CGTGGAGGTGCAGTGGGA(R)CC(N)TT)3'; P2, 5'-GGCGGAGATTCCGACTCC(R)TA(Y)TC(N)GT-3')] based

\section{Received March 31, 2003; revised May 13, 2003; accepted May 16, 2003.}

This work was supported by Deutsche Forschungsgemeinschaft Grant Be1654/3. We thank Drs. Udo Bartsch, Chi-Bin Chien, and Lara Hutson for helpful discussions. We are grateful to Laura Sander for correcting the English and Angelika Nest for excellent fish care.

Correspondence should be addressed to Dr. Catherina G. Becker, Zentrum für Molekulare Neurobiologie Hamburg, Universität Hamburg, Martinistrasse 52, D-20246 Hamburg, Germany. E-mail: tcbecker@zmnh.unihamburg.de.

Copyright $\odot 2003$ Society for Neuroscience $\quad$ 0270-6474/03/236232-06\$15.00/0 on the conserved fibronectin type III (FN III)-like domains of human, rat, and chicken tenascin-R using the Consensus-Degenerate Hybrid Oligonucleotide Primer program (Rose et al., 1998). A degenerate 5' oligonucleotide primer [P3, 5'-GCCTTTCGAGTGCCGG(Y)T(N)GA(R)GT(N)AC-3'] and a gene-specific 3' primer (PS3, 5' -CCACTCCACAAACGCAACCGTGT- $3^{\prime}$ ) located in the most $5^{\prime}$ end of the cloned tenascin- $\mathrm{R}$ fragment were used to clone a second gene fragment. To clone the complete gene, $5^{\prime}$ rapid amplification of cDNA ends (RACE) and 3' RACE were performed using the appropriate kits (Invitrogen, Karlsruhe, Germany).

Northern blot analysis. Northern blot analysis of RNA from adult zebrafish brain has been described previously (Schweitzer et al., 2003). An $\alpha-{ }^{32} \mathrm{P}$-labeled tenascin-R cRNA probe, $500 \mathrm{bp}$ in length, was generated by PCR using adult brain cDNA as template (N1, 5'-GGTGATTGACAGCGACATTA-3'; N2, 5'-GTCCTTGGTAGAGAAAGG-3'). The tenascin-C probe has been described previously (Weber et al., 1998).

Morpholino injections and analysis of the trajectories of optic axons. Two morpholinos of non-overlapping sequence (morpholino1, GCACCCCCACATCACAACTCGGGC; morpholino2, CATCAACTCCACAAATAGACCTCTG) binding to the $5^{\prime}$ untranslated region (UTR) of zebrafish tenascin-R mRNA were purchased from GeneTools (Corvallis, OR). As a specific control, a morpholino with a sequence similar to morpholino1, in which four bases were mismatched, was injected (control morpholino, GCtCCgCCACATCACAACaCGcGC). Morpholinos were diluted to $1 \mathrm{~mm}$ in Danieau solution (Nasevicius and Ekker, 2000) and injected into fertilized eggs $(0.5-1 \mathrm{nl} / \mathrm{egg})$.

To label a part of the optic projection, 3-d-old larvae were anesthetized, fixed in $4 \%$ paraformaldehyde overnight, washed, and embedded in low melting agarose (1\%). A glass needle covered with DiI (Molecular Probes, Eugene, OR) was inserted into the temporal retina for $20 \mathrm{sec}$. After incubation at room temperature overnight, the contralateral eye was removed, larvae were whole mounted on the injected side, and the optic projection was viewed using a laser scanning microscope (Zeiss, Oberkochen, Germany). DiI was confined to the temporal half of the retina. Thirty to 40 optical sections ( $2 \mu \mathrm{m}$ in thickness), comprising the entire optic projection in the brain, were examined (see Fig. 4). 
Optic axons enter the brain ventrally through the chiasm (see Fig. 2 A). Most axons run tightly fasciculated through the optic tract toward the optic tectum, in which they arborize superficially in a retinotopic manner (see Fig. 2A) (Stuermer, 1988). Along the optic tract, we observed several arborization fields, which are clusters of thin, beaded and randomly branched fibers as described by Burrill and Easter (1994). Most arborization fields are located medial of the optic tract. In our observations, the fields were only visible in single optical sections. They were obscured in image stacks viewed from a lateral perspective. Arborization field 3 of Burrill and Easter (1994) is located 75-125 $\mu \mathrm{m}$ caudal to the optic tract and is, therefore, visible in image stacks (see Fig. $4 A$ ).

We scored the number of axonal branches that grew horizontally away from the optic tract for $>135 \mu \mathrm{m}$, either "overshooting" arborization field 3 or growing parallel to it. These branches were defined as excessive, because they were not associated with any arborization field. The branches may contain one or several axons, which cannot be resolved in the confocal images. Larvae were scored as showing excessively branched optic tracts only when their labeled tract contained more than one branch longer than $135 \mu \mathrm{m}$. This is a conservative measure to ensure that one branch growing to arborization field 3 is not mistaken for being excessive. In the tectum, we analyzed whether axons were located in retinotopically inappropriate caudal positions and whether they grew from their normal superficial position into deeper layers in confocal images. To depict at low magnification the entire projection with thin axon branches leaving it, composite drawings of individual confocal images from a computer screen (see Fig. 4C,D) were used.

Western blot analysis. Western blot analysis using a rabbit polyclonal antibody to the epidermal growth factor (EGF)-like repeats and the $\mathrm{N}$-terminal cysteine-rich region of mouse tenascin-R (anti-EGF-L) (Xiao et al., 1998), which specifically reacts with tenascin-R of zebrafish (see Results), was done as described previously (Becker et al., 2000). For immunoblots of morpholino-treated larvae, detergent lysates of five heads of 3-d-old larvae were pooled for each lane and separated on an $8 \%$ polyacrylamide gel. Stripping and reprobing of blots with an antibody to tubulin (6-11B-1; Sigma, Deisenhofen, Germany) indicated that equal amounts of protein were loaded.

In situ hybridization. Digoxigenin-labeled RNA sense and antisense probes for tenascin-R mRNA were generated from a $700 \mathrm{bp}$ fragment located within the FN III-like domains or a $3.2 \mathrm{~kb}$ C-terminal fragment containing the 3' UTR using the Megascript kit (Ambion, Huntingdon, UK). Nonradioactive detection of mRNAs in tissue sections (14 $\mu \mathrm{m}$ in thickness) was performed as described previously (Becker et al., 2000). Both antisense probes showed identical results. Sense probes, developed in parallel under the same conditions, did not show any labeling (see Fig. $3 F$ ).

Immunohistochemistry. Immunolabeling of cryosections, $14 \mu \mathrm{m}$ in thickness, of fresh frozen larvae was performed as described previously (Becker et al., 2000). To reveal optic axons, we used the mouse monoclonal linc antibody (Developmental Studies Hybridoma Bank, Iowa City, IA) (Steen et al., 1989) to a neurofilament-associated antigen. This antibody labels optic axons in zebrafish, as deduced from comparison with axonal tracing. Other long axon fascicles, such as the posterior commissure, were also labeled by the antibody. Because these fascicles were remote from the optic projection, they could not be confused with the latter. For double immunohistochemistry, the anti-EGF-L and the linc antibody were applied simultaneously and detected with the appropriate Cy2- or Cy3-coupled secondary antibodies (Dianova, Hamburg, Germany).

To semiquantitatively assess expression of tenascin- $\mathrm{R}$ in sections of morpholino-injected animals, photomicrographs were taken with a digital camera mounted on a fluorescence microscope (Zeiss) using the same exposure times. Intensity profiles of tectal fiber layers were analyzed using the Zeiss LSM510 software. Fluorescence intensity values were averaged for three larvae (two sections per larva) per treatment. Maximal intensities in deep tectal fiber layers of uninjected animals were defined as $100 \%$. Fluorescence intensity in adjacent unlabeled fiber layers was defined as baseline and subtracted from the measurements.

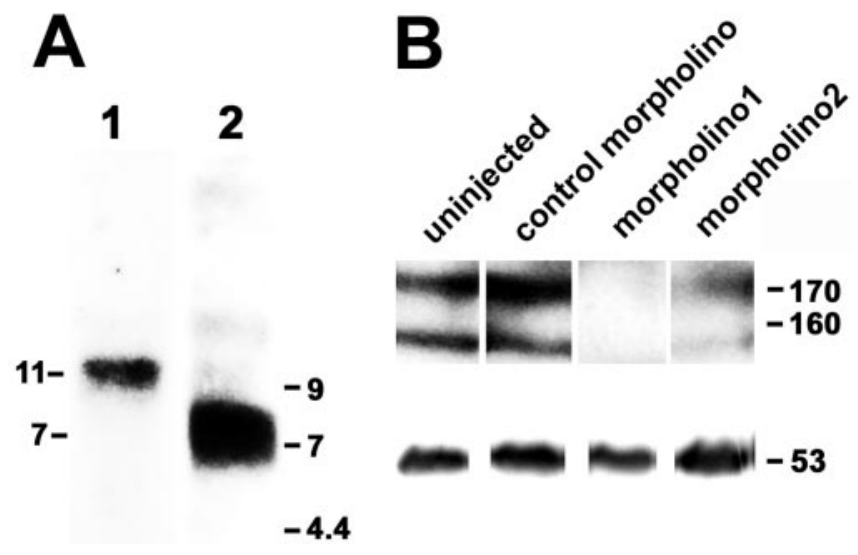

Figure 1. Tenascin-R mRNA and protein are expressed in zebrafish. $A$, Northern blot analysis of total RNA isolated from adult zebrafish brains yielded a band at $11 \mathrm{~kb}$ (lane 1). A probe to tenascin-C labeled a band at $7 \mathrm{~kb}$ (lane 2). Lengths of transcripts are indicated on the left; positions of molecular markers are indicated on the right. $B$, Western blot analysis using antiEGF-L antibody on detergent lysates of heads of 3-d-old larvae, separated on the same gel, indicate reduction of immunoreactivity by morpholino1 and morpholino2 but not by control morpholino. Reprobing of the blot with an antibody to tubulin is depicted in the bottom row. Molecular weights of tenascin- $R$ and tubulin are indicated on the right.

\section{Results}

Cloning and expression of tenascin- $\mathrm{R}$ in zebrafish

The complete open reading frame of tenascin- $\mathrm{R}$ encodes a deduced protein of 1351 amino acids (Genbank accession number AY286489). The start codon was predicted from the presence of stop codons preceding the N-terminal end of the deduced protein and of a signal peptide at the $\mathrm{N}$ terminus. The domain structure of zebrafish tenascin-R comprising a cysteine-rich domain, 4.5 EGF repeats, 9 FN III-like domains, and 1 fibrinogen globe at the $\mathrm{C}$ terminus, is identical to that of homologs in other vertebrates (Jones and Jones, 2000). The amino acid sequence of zebrafish tenascin- $\mathrm{R}$ is $\sim 60 \%$ identical to those of tenascin- $\mathrm{R}$ in chicken, human, and rat (http://www.ncbi.nlm.nih.gov/BLAST). A transcript of $11 \mathrm{~kb}$, which is similar in length to the $12 \mathrm{~kb}$ tenascin- $\mathrm{R}$ transcript found in adult mouse brain tissue (Weber et al., 1999), was found in adult zebrafish brain by Northern blot analysis. Reprobing the blot with a probe to tenascin-C indicated a size of $7 \mathrm{~kb}$ for the transcript (Weber et al., 1998), suggesting specificity of the tenascin-R signal (Fig. $1 A$ ).

Western blot analysis with anti-EGF-L revealed characteristic double bands at $\sim 160$ and $170 \mathrm{kDa}$ for tenascin-R (160 and 180 in mice) (Bartsch et al., 1993) in detergent lysates from heads of 3-d-old larvae (Fig. $1 B$ ) and adult brains (data not shown). Injecting $1 \mathrm{~mm}$ morpholino1 or morpholino2 against tenascin-R into fertilized eggs reduced protein labeling to undetectable (morpholino1) or lower levels (morpholino2) compared with control morpholino-injected or uninjected larvae. We conclude that the anti-EGF-L antibody recognizes the two tenascin-R isoforms in zebrafish and that tenascin-R mRNA and protein are present in the CNS of zebrafish.

\section{Tenascin-R borders the developing optic projection}

Optic fibers first establish retinotopic innervation of the tectum at $3 \mathrm{~d}$ of development (Fig. 2A,B) (Stuermer, 1988). To analyze the relationship between tenascin-R and optic axons, we simultaneously labeled optic axons with the linc antibody and tenascin-R with the anti-EGF-L antibody at 3-5 d of development (Fig. $2 C-K$ ). In the retina, the outer plexiform layer was conspicuously labeled for tenascin-R. This evolutionarily con- 
served pattern of protein labeling and of corresponding mRNA expression in the adjacent inner nuclear layer, most likely corresponding to horizontal cells (see below), lays separate from the optic fiber layer (Bartsch et al., 1993). Its functional significance is unknown. We used it here as a control for the specificity of tenascin-R mRNA and protein detection. The linc-immunoreactive retinal optic fiber layer, the optic nerve (data not shown), and tract were tenascin-R immunonegative (Fig. 2C, $D, F-K$ ). In crosssections through the diencephalon, tenascin- $\mathrm{R}$ immunoreactivity borders the chiasm and optic tract medially (Fig. $2 \mathrm{~F}-$ $H)$. In sagittal sections, tenascin-R immunoreactivity can be seen to border the tract also caudally but not rostrally, where the diencephalic periventricular cell layer is located (Fig. 2 $I-K$ ) (Wilson et al., 1990). Thus, tenascin-R could prevent optic axons from leaving the optic tract in a mediocaudal direction. In the tectum, deep fiber layers were more strongly labeled than the superficial optic neuropil (Fig. 2E). This may prevent optic axons from invading deeper tectal layers.

Tenascin- $\mathrm{R}$ mRNA was intensely labeled in one band of cells located at the outer margin of the inner nuclear layer of the retina, which is consistent with the location of horizontal cells, at 3-5 d of development (Fig. 3E,F). Synthesis of tenascin- $\mathrm{R}$ in these cells could lead to deposition of this secreted extracellular matrix molecule via axonal cell surface receptors in the adjacent outer plexiform layer (Fig. $3 A, B$ ). No tenascin-R mRNA was detectable in the optic nerve or tract, but it was found in scattered cells in the periventricular cell layer throughout the diencephalon and tectum (Fig. 3E,F). This mRNA expression could be related to the widespread immunoreactivity in deep fiber layers of the diencephalon and tectum.

\section{Reducing tenascin-R expression increases caudal branching of the optic tract}

To see whether morpholino treatment reduces tenascin- $\mathrm{R}$ immunoreactivity in the brain, relative fluorescence intensities in the deep tectal fiber layer were analyzed in 3-d-old larvae (see Materials and Methods). Morpholinol reduced the intensity of immunolabeling in the brain to $9 \pm 2.3 \%$ (SEM) of that in uninjected controls $(100 \pm 5.8 \%)$. Morpholino2 reduced labeling to $28 \pm 7.8 \%$, whereas control morpholino had no effect (99 \pm $6.0 \%)$. This confirms results from Western blots, which indicated that morpholino1 was more efficient than morpholino2. Morpholino treatment also reduced labeling in the retinal outer plexiform layer (Fig. 3A-D). In 5-d-old larvae injected with morpholino1, immunoreactivity was increased again (data not shown).

We labeled optic axons from the temporal retina with DiI to detect an effect of the lack of tenascin-R on their projection pat- tern. Axons were strongly fasciculated in the ventral optic tract, branched into the optic radiation in the dorsal diencephalon, and terminated in the retinotopically appropriate rostral tectum in uninjected and control morpholino-injected 3-d-old larvae (Fig. $4 A, C)$. Axons were consistently observed to branch off the strongly fasciculated ventral part of the optic tract caudally to form arborization field 3 of Burrill and Easter (1994) (see Materials and Methods) in confocal image stacks (Fig. 4A). Occasionally, one or more branches of the optic tract, each containing one or more axons, grew caudally away from the optic tract and beyond arborization field 3 (at least $135 \mu \mathrm{m}$ away from the main optic tract). Such branches have not been reported previously in zebrafish (Burrill and Easter, 1994). We defined these as excessive branches (see Materials and Methods). The incidence of excessive branches was increased in morpholino-injected larvae (Fig. $4 B, D)$. 

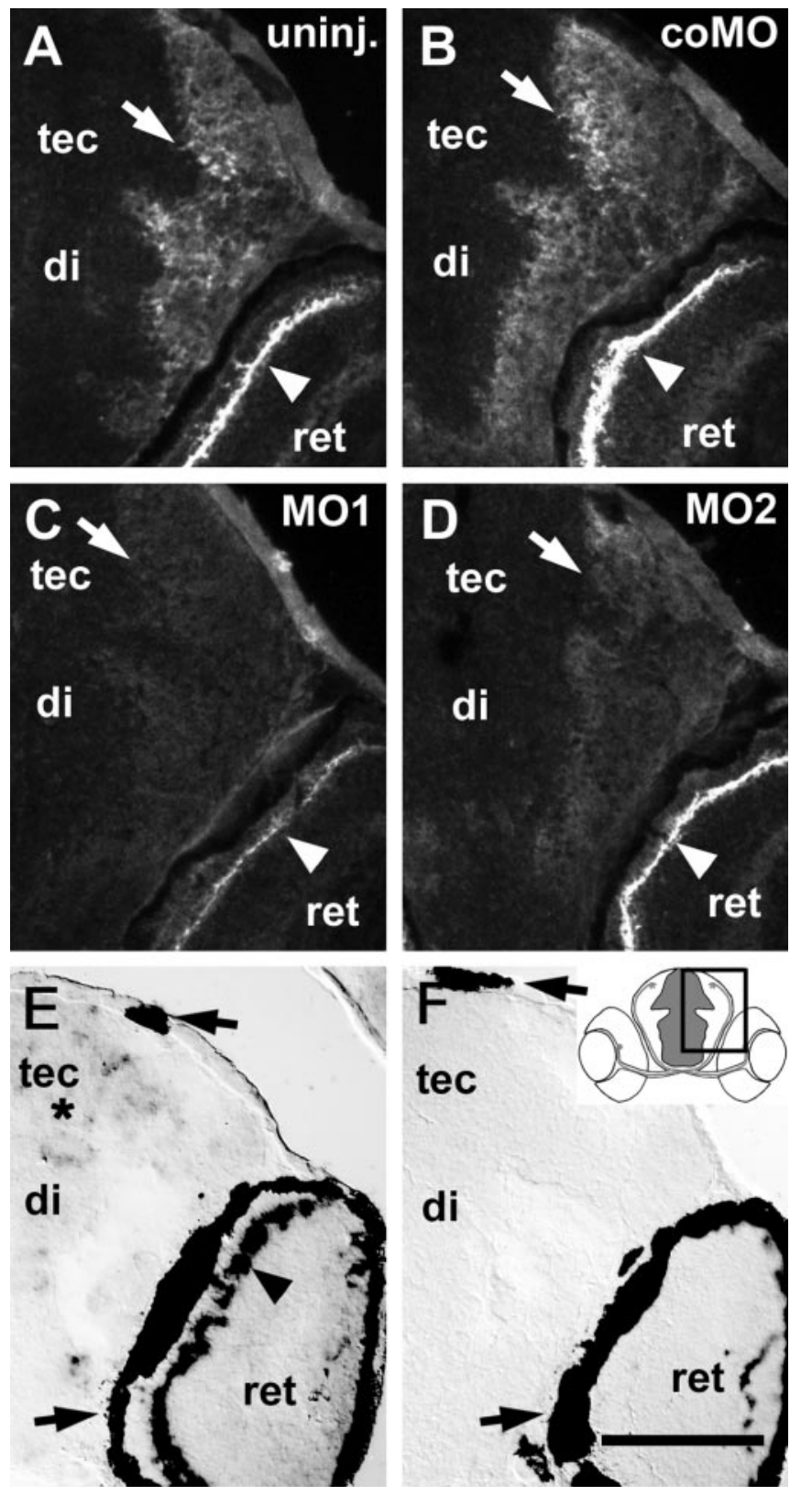

Figure 3. Effect of morpholinos on tenascin-R immunoreactivity and tenascin-R mRNA expression in 3-d-old larvae. Cross-sections including the diencephalon (di), tectum (tec), and part of the retina (ret) correspond to the boxed area in the inset in F. A-D, Arrows depict deep tectal fiber layers, and arrowheads indicate the outer plexiform layer of the retina. In uninjected $(A$, uninj.) and control morpholino-injected ( $B$, coMO) larvae, tenascin- $R$ immunoreactivity is most intense in the outer plexiform layer of the retina and high in deep fiber layers of the tectum and diencephalon. Injection of morpholino1 (C, M01) more strongly reduces immunoreactivity than morpholino2 (D, M02). E, F, Tenascin-R mRNA is detected by in situ hybridization in scattered cells throughout the periventricular cell layer of the brain (asterisk) and in a row of cells in the retina (arrowhead in $E$ ) but not after incubation with a sense RNA probe $(F)$. Arrows in $E$ and $F$ indicate the retinal pigment epithelium and pigment cells in the epidermis, which always appear black. Scale bar (in F): $A-F, 100 \mu \mathrm{m}$.

We quantified the number of larvae with excessively branched optic tracts, defined as tracts with at least two excessive branches (see Materials and Methods). Morpholino1 induced a statistically significant (Fisher's exact test) increase in the proportion of larvae with excessively branched optic tracts $(86 \% ; 12$ of 14 larvae) compared with uninjected (37\%; 5 of 14 larvae; $p=0.001)$ and control morpholino-injected (20\%; 4 of 20 larvae; $p=0.0006)$ larvae (Fig. 4E). There was no statistically significant difference between uninjected and control morpholino-injected larvae $(p>0.05)$. The proportion of animals with excessively branched tracts was also slightly increased after injection of morpholino2 (44\%; 7 of 16 larvae; not significant; $p>0.05$ ).

The number of excessive branches per animal was also significantly increased (Mann-Whitney $U$ test) after injection of morpholino1 (4.0 \pm 1.19 fascicles per larva) compared with uninjected $(1.7 \pm 0.65$ fascicles per larva; $p=0.019)$ and control morpholino-injected $(1.3 \pm 0.51$ fascicles per larva; $p=0.001)$ larvae (Fig. $4 F$ ). Values for uninjected and control morpholinoinjected larvae did not show statistically significant differences $(p>0.05)$. Morpholino 2 again showed a tendency toward increased numbers of excessive branches $(2.5 \pm 0.87$ fascicles per larva), but values did not reach statistical significance ( $p>0.05)$. Notably, almost all excessive branches that exited from the optic tract along its entire dorsoventral extent grew caudal (52 of 56 branches in morpholinol-injected larvae).

In addition to the optic tract, we analyzed the optic projection from the optic nerve through the chiasm up to the tectum using confocal microscopy. Axons in the chiasm always appeared tightly fasciculated. In the tectum, labeled axons were found in superficial fiber layers confined to the rostral tectum in morpholino-treated and control larvae (Fig. 4C,D), suggesting that growth in the chiasm, as well as layer-specific growth and retinotopy of optic axons in the tectum, were not influenced by the morpholino treatment.

\section{Discussion}

The conserved domain structure, high degree of amino acid identity, and conserved mRNA expression pattern in the inner nuclear layer of the retina strongly suggest that we cloned a zebrafish homolog of tenascin-R. Reducing expression of tenascin-R using morpholinos increased caudal branching of the optic tract in 3-d-old larvae. The incidence of larvae with excessively branched optic tracts was $86 \%$ compared with 20 and $37 \%$ in control morpholino-injected and uninjected larvae, respectively. The number of excessive branches of the optic projection more than doubled in experimental larvae (4.0 vs $1.3-1.7$ fascicles per larva in controls). The specificity of this effect is suggested by several observations. Injection of a control morpholino, in which 4 of 24 bases had been exchanged, did not show a significant effect on branching. In larvae injected with a second morpholino (morpholino2) of non-overlapping sequence with the first morpholino (morpholino1), the proportion of larvae with excessively branched tracts (44\%), as well as the average number of excessive branches, was also increased (2.5 fascicles per larva). However, the effect was smaller than that of morpholinol and did not reach statistical significance. This is probably because morpholino2 is not as efficient as morpholinol in reducing protein expression, as demonstrated by Western blot and immunohistochemistry. The difference between the two morpholinos suggests a specific, dosedependent effect of tenascin-R. Moreover, the pattern of tenascin-R immunoreactivity correlates with the direction of the additional branches observed. Tenascin-R borders the optic tract mediocaudally but not rostrally, and excessive branches were observed almost exclusively in the caudal direction after reduction of tenascin-R expression. Excessive medial branches were not observed, suggesting that, in the absence of tenascin- $\mathrm{R}$, axons may follow unidentified secondary guidance cues caudally. In uninjected control embryos, excessive caudal branches of the optic projection were occasionally also observed ( 1.7 fascicles per larva). In a previous study, these fibers have not been found after 
tracing with horseradish peroxidase and analysis by conventional light microscopy (Burrill and Easter, 1994). Tracing with DiI, in combination with the confocal microscopy used in this study, may be a more sensitive method. Thus, the border-like expression pattern in the ventral diencephalon and the pathfinding errors committed by optic axons in vivo after reduction of tenascin-R expression suggest a repellent guidance function of the molecule for optic axons in the developing optic tract.

Additional repellent guidance cues probably cooperate with tenascin- $\mathrm{R}$ to confine the optic tract. For example, rostral to the optic tract, in which tenascin-R is absent, mRNA of the axon-repellent extracellular matrix molecule slit3 is detectable by in situ hybridization. In addition, mRNA for a second member of the slit family (slit2) is expressed rostral and caudal to the tract (Hutson and Chien, 2002). Indeed, a mutant zebrafish not expressing Robo2, a receptor for slits, shows misrouting of optic axons in the ventral diencephalon (Fricke et al., 2001). Slit-deficient mice show similar phenotypes (Plump et al., 2002). Repellent semaphorins anterior to the optic tract may also play a role in guiding optic axons in Xenopus (Campbell et al., 2001). Expression of additional repellent guidance cues or residual expression of tenascin- $\mathrm{R}$ in morpholino-treated larvae could explain why misrouting of optic axons into deeper tectal layers, which normally contain higher levels of tenascin- $\mathrm{R}$ than the superficial optic neuropil, was not observed. In summary, our analysis shows that tenascin- $\mathrm{R}$ is involved in pathfinding of optic axons in the optic tract, probably as a contact-repellent guidance molecule.

\section{References}

Bartsch U, Pesheva P, Raff M, Schachner M (1993) Expression of janusin (J1-160/180) in the retina and optic nerve of the developing and adult mouse. Glia 9:57-69.

Becker T, Anliker B, Becker CG, Taylor J, Schachner M, Meyer RL, Bartsch U (2000) Tenascin-R inhibits regrowth of optic fibers in vitro and persists in the optic nerve of mice after injury. Glia 29:330-346.

Burrill JD, Easter SS (1994) Development of the retinofugal projections in the embryonic and larval zebrafish (Brachydanio rerio). J Comp Neurol 346:583-600.

Campbell DS, Regan AG, Lopez JS, Tannahill D, Harris WA, Holt CE (2001) Semaphorin 3a elicits stage-dependent collapse, turning, and branching in Xenopus retinal growth cones. J Neurosci 21:8538-8547.

Dickson BJ (2002) Molecular mechanisms of axon guidance. Science 298:1959-1964

Fricke C, Lee JS, Geiger-Rudolph S, Bonhoeffer F, Chien CB (2001) Astray, a zebrafish roundabout homolog required for retinal axon guidance. Science 292:507-510.
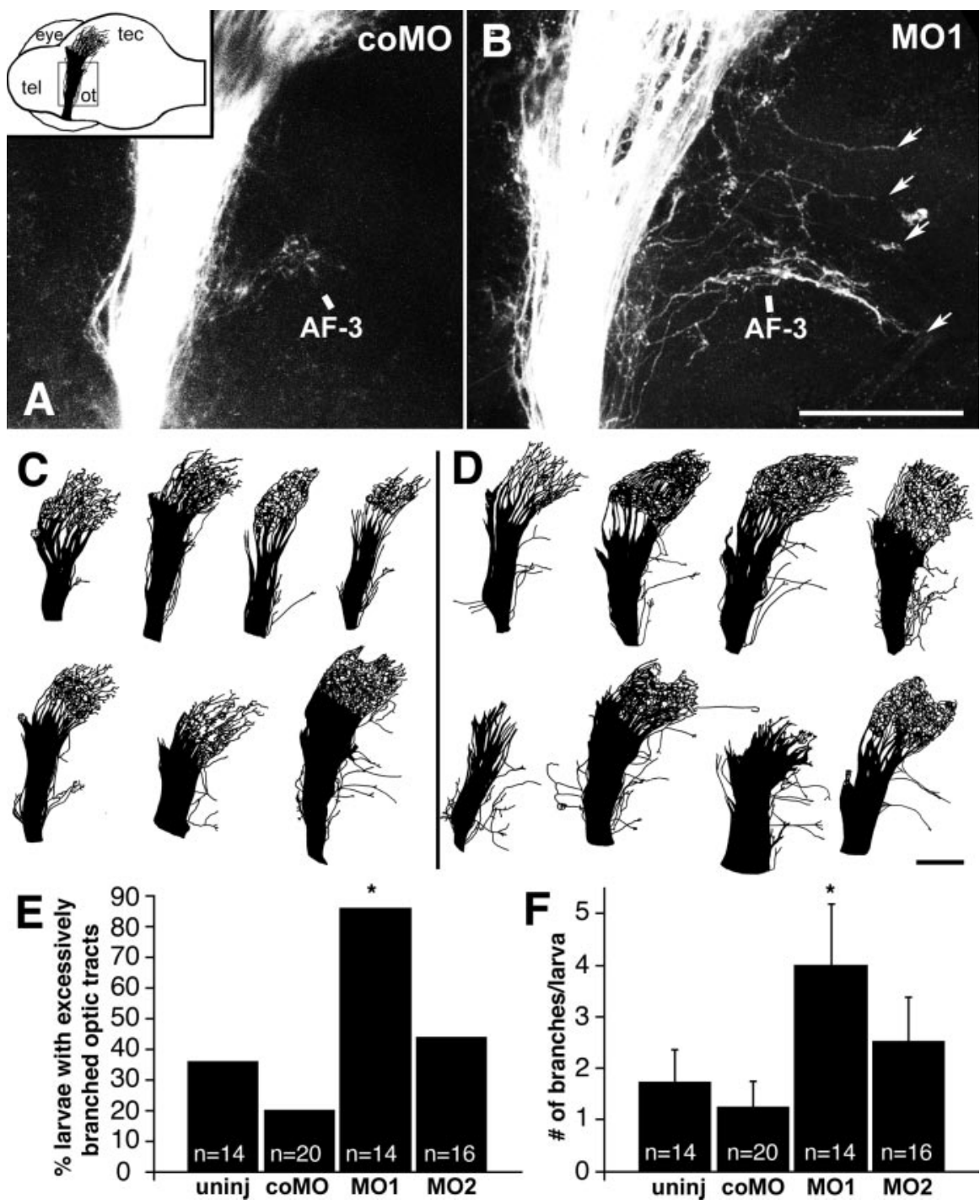

Figure 4. Morpholino treatment leads to excessive caudal branching of the optic tract in 3-d-old larvae. $A, B$, Lateral views of confocal image stacks of the optic tract in whole-mounted brains, after labeling of the temporal retina with Dil, correspond to the boxed area in the inset in $A$. The inset gives the orientation of the brain with the labeled optic projection indicated in black (tel, cephalon; ot, optic tract; tec, tectum; eye, contralateral eye; the ipsilateral eye was removed before mounting); dorsal is up,

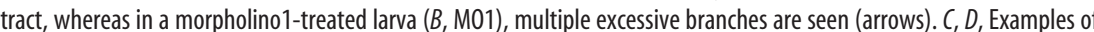

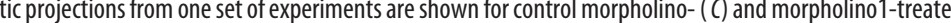
ences from controls are indicated by asterisks ( $E$, Fisher's exact test, $p \leq 0.001 ; F$, Mann-Whitney $U$ test, $p \leq 0.019$ ). Scale bars: (in $B$ ) $A, B, 210 \mu \mathrm{m}$; (in D) $C, D, 210 \mu \mathrm{m}$.

Hutson LD, Chien CB (2002) Pathfinding and error correction by retinal axons: the role of astray/robo2. Neuron 33:205-217.

Jones FS, Jones PL (2000) The tenascin family of ECM glycoproteins: structure, function, and regulation during embryonic development and tissue remodeling. Dev Dyn 218:235-259.

Kimmel CB, Ballard WW, Kimmel SR, Ullmann B, Schilling TF (1995) Stages of embryonic development of the zebrafish. Dev Dyn 203:253-310.

Nasevicius A, Ekker SC (2000) Effective targeted gene "knockdown" in zebrafish. Nat Genet 26:216-220.

Pesheva P, Gennarini G, Goridis C, Schachner M (1993) The F3/11 cell adhesion molecule mediates the repulsion of neurons by the extracellular matrix glycoprotein J1-160/180. Neuron 10:69-82.

Plump AS, Erskine L, Sabatier C, Brose K, Epstein CJ, Goodman CS, Mason CA, Tessier-Lavigne M (2002) Slit1 and Slit2 cooperate to prevent premature midline crossing of retinal axons in the mouse visual system. Neuron 33:219-232. 
Rose TM, Schultz ER, Henikoff JG, Pietrokovski S, McCallum CM, Henikoff S (1998) Consensus-degenerate hybrid oligonucleotide primers for amplification of distantly related sequences. Nucleic Acids Res 26:1628-1635.

Schachner M, Taylor J, Bartsch U, Pesheva P (1994) The perplexing multifunctionality of janusin, a tenascin-related molecule. Perspect Dev Neurobiol 2:33-41.

Schweitzer J, Becker T, Becker CG, Schachner M (2003) Expression of protein zero is increased in lesioned axon pathways in the central nervous system of adult zebrafish. Glia 41:301-317.

Steen P, Kalghatgi L, Constantine-Paton M (1989) Monoclonal antibody markers for amphibian oligodendrocytes and neurons. J Comp Neurol 289:467-480.

Stuermer CA (1988) Retinotopic organization of the developing retinotectal projection in the zebrafish embryo. J Neurosci 8:4513-4530.
Taylor J, Pesheva P, Schachner M (1993) Influence of janusin and tenascin on growth cone behavior in vitro. J Neurosci Res 35:347-362.

Weber P, Montag D, Schachner M, Bernhardt RR (1998) Zebrafish tenascin-W, a new member of the tenascin family. J Neurobiol 35:1-16.

Weber P, Bartsch U, Rasband MN, Czaniera R, Lang Y, Bluethmann H, Margolis RU, Levinson SR, Shrager P, Montag D, Schachner M (1999) Mice deficient for tenascin-R display alterations of the extracellular matrix and decreased axonal conduction velocities in the CNS. J Neurosci 19:4245-4262.

Wilson SW, Ross LS, Parrett T, Easter SS Jr (1990) The development of a simple scaffold of axon tracts in the brain of the embryonic zebrafish, Brachydanio rerio. Development 108:121-145.

Xiao ZC, Revest JM, Laeng P, Rougon G, Schachner M, Montag D (1998) Defasciculation of neurites is mediated by tenascin-R and its neuronal receptor F3/11. J Neurosci Res 52:390-404. 